\section{Limber tail syndrome in German shepherd dog}

\author{
Ghazanfar Abbas, 1 Muhammad Saqib, 1 \\ Mudassar Niaz Mughal, 1 Asif Ali Butt,2 \\ Ghulam Muhammad1 \\ 1Department of Clinical Medicine and \\ Surgery, Faculty of Veterinary Sciences, \\ University of Agriculture, Faisalabad; \\ 2Civil Veterinary Hospital, Faisalabad, \\ Pakistan
}

\begin{abstract}
A 3-year-old intact female German shepherd dog weighing $25 \mathrm{~kg}$ was evaluated for acute onset of staggering gait and flaccid (dropped) tail following immersion in freeze water. Clinical signs included staggering gait, aggressive behavior, postural pain and flaccid tail. Clinical signs progressed in severity for 2-3 hours following immersion in freeze water. The dog was treated using anti-inflammatory medication along with warm fomentation of the affected portion of the tail and complete rest. Dog uneventfully recovered on day 7 of initiation of treatment and no reoccurrence was observed during one month follow up period. Limber tail syndrome is an uncommonly reported condition in German shepherd dogs that should be considered when sudden staggering gait and dropped tail is observed after immersion in too cold water. Recovery is possible with anti-inflammatory drugs and rest.
\end{abstract}

\section{Introduction}

Limber tail syndrome (LTS) is an acute caudal (coccygeal) myopathy which is also known as cold tail, water tail, limp tail, swimmer tail, frozen tail, flaccid tail and sprain tail. This condition usually affects working, sporting and hunting dogs including English Pointers, Dalmatian, Beagles, Golden Retrievers, Setter, Foxhounds and Labrador Retrievers. There is no sex predisposition and condition is seen equally in both male and female dogs. The LTS is characterized by either hanging of the tail from its base or strutting out dorsally (in a horizontal direction) for many inches from the tail base followed by hanging ventrally without tone giving the appearance of broken tail.1-3 The exact etiology is still unclear but several factors including exposure to too cold or too warm climate, prolonged cage transportation, vigorous exercise (hunting and swimming) are thought to be associated with the develop- ment of this condition..$^{3,4}$ All these factors results in the overexertion of the muscles (especially intertransversarius ventralis muscle) resulting ischemic injury and development of limber tail syndrome. ${ }^{3,4}$ It has also been reported that LTS develops as a result of inflammation of the tendons and muscles surrounding the sacrococcygeal region. 5 The present report documents a first case of LTS in a female German shepherd dog.

\section{Case Report}

A 3-year-old intact female German shepherd dog weighing $25 \mathrm{~kg}$ was referred to Veterinary Medical Teaching Hospital, Department of Clinical Medicine and Surgery (CMS), University of Agriculture Faisalabad, Pakistan, for the treatment of acute onset of staggering gait, aggressive behavior, postural pain and flaccid (dropped) tail following immersion in freeze water (Figure 1A). Anamnesis indicated that the patient was given a bath (immersed in freeze water) to reduce temperature and panting that had developed subsequent to prolonged exposure to direct sun light. Clinical examination revealed normal rectal temperature $\left(39^{\circ} \mathrm{C}\right)$, respiration rate $(25$ breaths/minute) and heart rate (120 beats/minute). Musculoskeletal and neurologic examination findings were insignificant and patient had intact nociception, however severe pain reflex was noted at the base of tail. Radiographic and hematologic examinations were also normal, nevertheless, serum biochemistry revealed slightly elevated level of creatine kinase CK (Tables 1 and 2). On the basis of history, clinical examination and an elevated serum CK level, a diagnosis of limber tail syndrome was reached.

\section{Treatment}

In view of the nature of injury in limber tail, the patient was treated with the combination of ketoprofen $(2.2 \mathrm{mg} / \mathrm{kg})$ and dexamethasone $(0.5 \mathrm{mg} / \mathrm{kg})$ intramuscularly once a day and discharged with an advice to the owner to provide complete rest to the patient and application of warm fomentation at the base of tail. On day 2 , the treatment was repeated since patient still had pain and weakness of hind legs. On 3rd day of treatment, a marked reduction in the severity of pain was noticed, however, the patient still had a dropped tail (Figure 1B) and developed anorexia. Hemato-biochemical values were within normal ranges (Tables 1 and 2). Ranitidine (2 mg/kg) was added to the treatment protocol for next 3 days. The patient completely recovered on day 7 of treatment, tail returned to its normal position (Figure 1C) and had no pain reflex on examination. Patient also regained its normal feed
Correspondence: Ghazanfar Abbas, Department of Clinical Medicine and Surgery, Faculty of Veterinary Sciences, University of Agriculture, Faisalabad, 38040, Punjab, Pakistan.

Tel: +92.332 .6385473 .

E-mail: ghazanfarabbas3479@gmail.com

Key words: Creatine phosphokinase; freeze water; German shepherd; limber tail.

Contributions: the authors contributed equally.

Conflict of interest: the authors declare no potential conflict of interest.

Received for publication: 28 January 2015. Accepted for publication: 28 February 2015.

This work is licensed under a Creative Commons Attribution NonCommercial 3.0 License (CC BYNC 3.0).

(C) Copyright G. Abbas et al., 2015

Licensee PAGEPress srl, Italy

Veterinary Science Development 2015; 5:5831 doi:10.4081/vsd.2015.5831

intake and recreational activities. Hemato-biochemical parameters were within normal limits. Ketoprofen was discontinued and dexamethasone was tapered over next one week. The dog did not experience any recurrence of syndrome during 1-month follow up period.

\section{Discussion and Conclusions}

The present case report describes cold water induced LTS in a female German shepherd dog. The clinical signs of LTS including staggering gait, severe pain at the base of tail and flaccid tail have been reported in working breeds of young dogs. 1.6 The precise pathogenesis is yet to determine, however histopathological investigations of affected parts of coccygeal muscles indicated lesions are compatible with ischemia. Similar lesions have been documented in human muscles associated with an acute compartmental syndrome. This syndrome was noted in muscles that relatively having thick layer of fascia. Stressful events lead to edema and subsequent ischemia owing to non compliant fascial sheath. ${ }^{3}$ This condition is frequently misdiagnosed as trauma associated fracture, spinal cord anomalies (intervertebral disc protrusion/extrusion, neoplasm, lumbrosacral syndrome etc) and prostatic diseases particularly in those regions where this condition is not well known. However, the hunting dog's trainers are well familiar to this condition and a rarely seek assistance of veterinarians as they know that the condition is self-resolving. In hunting dogs 
especially in Pointers, the chief predisposing factors that have been linked with this condition are transportation in cages for extended time duration and exposure to warm and cold weather. Contrarily, the Retrievers usually develop this condition after swimming and bathing in water. ${ }^{7}$ Coccygeal muscles particularly intertransversarius ventralis is severely affected in LTS which has a significant role for the proper functioning of tail. This muscle along with intertransversarius dorsalis is involved in lateral flexion of the tail. ${ }^{8}$ Lateral movement of tail is necessary for the maintenance of coordination and balance of the body during locomotion. ${ }^{9}$ If due to any reason this movement of the tail is affected, it would lead towards staggering gait. The incoordination of gait in the affected female German shepherd dog of our case study may be attributed to coccygeal muscle damage as the lateral movement of tail was not present. A slightly increased level of CK (an indicator of muscular injury) has been documented in limber tail patients that usually return to normal within 12-48 hours. Similar result were noted in our case report as slightly elevated values of $\mathrm{CK}$ at the time of presentation which returned to normal on re-examination after 24 hours. Most of the affected dogs recover spontaneously with or without any treatment within days to weeks; however few cases may show recurrence. ${ }^{10}$ The present report endorses the role of utiliza- tion of warm fomentation at the base of the tail and administration of anti-inflammatory in pain management to enhance recovery. ${ }^{1,4}$ To best of the author's knowledge, this first case report of LTS in German shepherd which is diagnosed by history, clinical picture, physical examination, radiographical findings, hematobiochemical reports and a fairly prompt recovery. As also, this indicates the risk of coccygeal muscles injury in German shepherd subsequent to cold exposure. However present communication lacks histopathology and electromyographic investigations, which need to be studied in German shepherd dogs.

Table 1. Hematological values of a female German shepherd suffering from limber tail syndrome.

\begin{tabular}{lcccc}
\hline & \multicolumn{3}{c}{ Values recorded } & Reference \\
& Day 1 & Day 3 & Day 7 & \\
Red blood cells, $\times 1012 \mathrm{~g} / \mathrm{L}$ & 6 & 5.50 & 5.01 & $5.5-8.5$ \\
Packed cell volume, \% & 40.2 & 37.9 & 34.8 & $37-55$ \\
\hline Hemoglobin, g/dL & 17 & 16.1 & 14.5 & $12-18$ \\
Mean corpuscular volume, fL & 72 & 69 & 69.5 & $60-77$ \\
\hline Mean corpuscular hemoglobin, pg & 21.5 & 24.4 & 25 & $19.5-24.5$ \\
Mean corpuscular hemoglobin concentration, $\times 10 \mathrm{~g} / \mathrm{L}$ & 34.8 & 36.9 & 35.6 & $32-36$ \\
\hline White blood cells, $\times 10^{9} \mathrm{~g} / \mathrm{L}$ & 12 & 10.5 & 9.5 & $6-17$ \\
Neutrophils, \% & 74.7 & 73.5 & 71.7 & $60-70$ \\
\hline Lymphocytes, \% & 20.3 & 20.5 & 21.3 & $12-30$ \\
Monocytes, \% & 4 & 5 & 5 & $4-10$ \\
\hline Basophils, $\%$ & - & - & - & Rare \\
Eosinophils, \% & 1 & 1 & 2 & $2-10$ \\
\hline
\end{tabular}

Table 2. Serum biochemistry values of a female German shepherd suffering from limber tail syndrome.

\begin{tabular}{|c|c|c|c|c|}
\hline \multirow[t]{2}{*}{ Parameters } & \multicolumn{3}{|c|}{ Values recorded } & \multirow{2}{*}{$\begin{array}{c}\text { Reference } \\
\text { Day } 7\end{array}$} \\
\hline & & Day 1 & Day 3 & \\
\hline Aspartate aminotransferase, $\mu / L$ & 25 & 26 & 30 & $8.9-49$ \\
\hline Alanine aminotransferase, $\mu / L$ & 33 & 31 & 36 & $8.2-57$ \\
\hline Alkaline phosphatase, $\mu / \mathrm{L}$ & 52 & 53 & 60 & $10.6-101$ \\
\hline Creatine kinase, $\mu / L$ & 140 & 21 & 17 & $14-120$ \\
\hline Total bilirubin, mg/dL & 0.3 & 0.3 & 0.3 & $0.1-0.6$ \\
\hline Creatinine, mg/dL & 1.4 & 1.3 & 1.3 & $0.5-1.6$ \\
\hline Blood urea nitrogen, mg/dL & 17 & 22 & 24 & $8.8-26$ \\
\hline Albumin, g/dL & 3 & 2.9 & 3 & $2.6-4.0$ \\
\hline Globulin, g/dL & 2.5 & 2.7 & 2.4 & $2.1-3.7$ \\
\hline Sodium, mmol/L & 125 & 118 & 120 & $140-154$ \\
\hline Potassium, mmol/L & 3.2 & 2.9 & 3.5 & $3.8-5.6$ \\
\hline Chloride, $\mathrm{mmol} / \mathrm{L}$ & 107 & 115 & 110 & $102-117$ \\
\hline Bicarbonate, mEq/L & 24 & 23 & 22 & $18-25$ \\
\hline
\end{tabular}

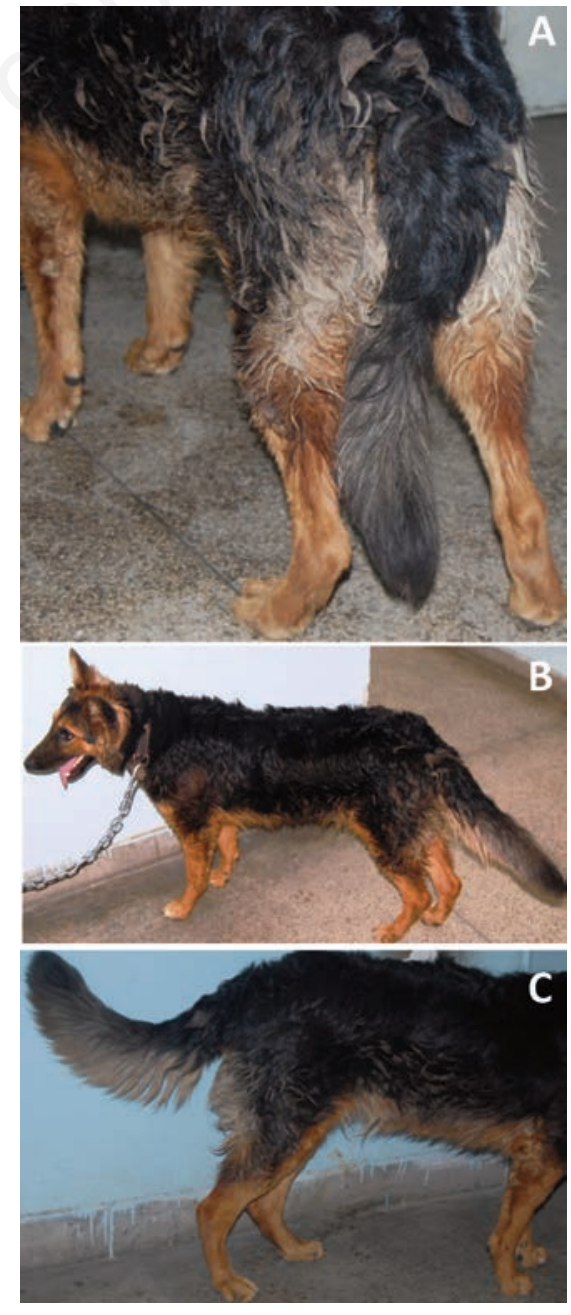

Figure 1. A) Flaccid tail of affected female dog showing typical sign of limber tail syndrome at day 1; B) the position of the tail at day 3 post initiation of treatment showing positive response to treatment; $\mathrm{C}$ ) position of the tail at day 7 post initiation of treatment showing normal position of tail. 


\section{References}

1. Steiss JE, Braund K, Wright J, Coccygeal muscle injury in English Pointers (limber tail). J Vet Inter Med 1999;13:540-8.

2. Dewey CW, Cerda-Gonzalaz S. Myopathies: disorders of skeletal muscles. In: Dewey $\mathrm{CW}$, ed. A practical guide to canine and feline neurology. $2^{\text {nd }}$ ed. Hoboken: WileyBlackwell; 2008.
3. Delahunta A, Glass E. Veterinary neuroanatomy and clinical neurology. 3rd ed. Philadelphia: W.B. Saunders Elsevier; 2009.

4. Stesis JE. Muscle disorders and rehabilitation in canine athletes. Vet Clin North Am Small Anim Pract 2002;32:276-85.

5. Wilkins CM. Frozen tail or limber tail inworking dogs. Vet Rec 1997;140:588.

6. Steiss JE, Braund KG. Frozen tail or limber tail in working dog. Vet Rec 1997;141:179.

7. Jeffels W. Frozen tail or limber tail in work- ing dogs. Vet Rec 1997;140:564.

8. Evans HE. Miller's anatomy of the dog. 3rd ed. Philadelphia: W.B. Saunders; 1999. p. 314.

9. Wada N, Hori H, Tokuriki M. Electromyographic and kinematic studies of the tail movements in dogs during treadmill locomotion. J Morph 1993;217: t103-5.

10. Hewison C. Frozen tail or limber tail in working dogs. Vet Rec 1997;140:536. 